\title{
New Insights in Treatment Options in Pediatric Urinary Tract Infection
}

\author{
Carmen Duicu1*, Iulia Armean², Cornel Aldea ${ }^{3}$ \\ 1. University of Medicine, Pharmacy, Sciences and Technology of Târgu Mureș, Târgu Mureș, Romania \\ 2. Pediatric Clinic 1, Emergency County Hospital Târgu Mureș, Romania \\ 3. Pediatric Nephrology Department, Emergency Clinical Hospital for Children Cluj-Napoca, Romania
}

Urinary tract infection (UTI) represents one of the most frequent infections with bacterial etiology during childhood. In infants and toddlers with fever without source UTI' investigation should be carried out, since signs and symptoms are nonspecific. However, obtaining uncontaminated urine samples from these patients can be challenging and time consuming; all current collection methods (clean-catch, plastic collection bag, catheterization, etc) have disadvantages. Criteria for UTI definition are represented by the presence of significant number of a single uropathogen, this number being different depending on the collection method: at least 1000 colony-forming unit (CFU/ml) for catheter samples and at least $100.000 \mathrm{CFU} / \mathrm{ml}$ from midstream clean-catch samples or $50.000 \mathrm{CFU} / \mathrm{ml}$ and significant pyuria in a symptomatic or febrile child. Accurate diagnosis of UTI is essential to avoid any antibiotic overuse and expensive investigations. UTI caused by resistant bacterial strains has an increasing prevalence in children. In pediatric population, extended spectrum beta-lactamase-producing Enterobacteriaceae (ESBLPE) represent the etiology of around 15\% of UTIs. Because of limited therapeutic options the reintroduction of some old antimicrobial agents is necessary, therefore Nitrofurantoin and Fosfomycin, can represent alternatives for oral treatment and prophylaxis of UTIs in children or in case of resistance suspicion to other drug classes. It is important to recognize patients at risk, such as children with recurrent UTIs, kidney abnormalities, like vesicoureteral reflux and previous antibiotherapy, in order to recommend adequate empiric treatment, especially against resistant bacteria.

Keywords: child, fever, urinary tract infection, treatment

Received 11 January 2019 / Accepted 10 February 2019

\section{Introduction}

Urinary tract infection (UTI) is considered as the second infections with bacteria in children. The diagnosis of UTI in infants and children can be difficult, as clinical picture varies with the patient's age, lack of characteristic symptoms in young infants, different collecting methods of urine sample, and interpreting the result of urinalysis and urine culture. In infants and toddlers with fever without source UTI' investigation should be carried out, since signs and symptoms are nonspecific. Careful anamnesis and complete physical examination are key-elements for the suspicion and diagnosis of UTI.

A detailed anamnesis to identify risk factors or an underlying renal pathology is essential (poor urine flow, dysfunctional voiding, enlarged bladder, previous UTI, recurrent fever without source, renal abnormality diagnosed antenatally, family history of renal disease, constipation, spine abnormalities, poor growth) (1).

Recently, many guidelines have tried to settle several debates in diagnosis of pediatric UTI. UTI is defined as a positive urine culture and urinalysis, plus clinical symptoms $(1,2)$. Significant pyuria is defined as $\geq 10$ leucocytes $/ \mathrm{mm}^{3}$ on an uncentrifuged urine sample or $\geq 5$ leucocytes/ high-power field on a centrifuged urine sample or presence of leukocyte esterase on urine dipstick (3). A positive nitrite test result is another argument for UTI, but a negative one has little value in ruling out UTI as it is not an accurate marker for infant' UTI, who urinate frequently and not all uropathogens reduce nitrate to nitrite (3).

\section{Clinical picture}

Neonates and young infants with febrile UTI are more likely to have bacteriemia or sepsis than older children and should be carefully evaluated and managed. UTI in newborn and infant can manifest as different "picture" and has non-specific symptoms (Table I).

\section{Laboratory}

To confirm a UTI, a proper collected urine sample for culture is mandatory, this being a challenge in non-toilet trained children. However, obtaining uncontaminated urine samples from these patients can be difficult and time consuming; all current collection methods (clean-catch, plastic collection bag, bladder catheterization and suprapubic aspiration) have disadvantages (Table II). We have to take into consideration that not all collection methods of the urine are equals! The final bacterial concentration is directly related to urine output, collection method, storage and shipment of the urine sample to laboratory $(1,3)$.

For many years the same cut-off value of $100.000 \mathrm{CFU} /$ $\mathrm{mL}$ of a single uropathogen has been the criteria for UTI diagnosis in schoolchildren and adults. It was based on the 
Table I. Clinical features in pediatric UTI based on patient' age

\begin{tabular}{|c|c|c|c|}
\hline Neonates, infants $<2$ months & $\begin{array}{l}\text { Infants and children aged } 2 \\
\text { months to } 2 \text { years }\end{array}$ & $\begin{array}{l}\text { Toddler, preschool children } \\
\text { ( } 2-6 \text { years) }\end{array}$ & $\begin{array}{l}\text { School-aged children, teenagers } \\
\text { (>6 years) }\end{array}$ \\
\hline $\begin{array}{l}\text { fever, prolonged jaundice, failure to } \\
\text { thrive, poor feeding, vomiting, diar- } \\
\text { rhea, irritability, lethargy } \\
\text { sepsis, hematuria, cloudy or mal- } \\
\text { odorous urine }\end{array}$ & $\begin{array}{l}\text { systemic symptoms: poor feeding, } \\
\text { high temperature, nausea and vomit- } \\
\text { ing, abdominal pain or discomfort, } \\
\text { irritability, malaise, strong-smelling } \\
\text { urine, hematuria, } \pm \text { crying on passing } \\
\text { urine }\end{array}$ & $\begin{array}{l}\text { gastrointestinal signs, such as vomiting and } \\
\text { diarrhea, abdominal pain; fever; } \\
\text { urinary symptoms: dysuria, frequency, } \\
\text { urgency, new onset urinary incontinence, } \\
\text { enuresis, abdominal or flank pain, supra- } \\
\text { pubic discomfort, strong-smelling urine, } \\
\text { hematuria, cloudy urine }\end{array}$ & $\begin{array}{l}\text { fever, vomiting, abdominal pain, flank/ } \\
\text { back pain with or without fever, urinary } \\
\text { symptoms (dysuria, urgency, frequent } \\
\text { voiding), enuresis, incontinence; suprapu- } \\
\text { bic pain, strong-smelling urine, hematuria, } \\
\text { cloudy urine }\end{array}$ \\
\hline
\end{tabular}

Table II. Disadvantages of urine specimen collection method

\begin{tabular}{ll}
\hline Collection method & Disadvantages \\
\hline Suprapubic aspiration & invasive method, painful \\
Bladder catheterization & the risk of introduction of nosocomial pathogens \\
Clean voided midstream urine sample & first morning sample \\
Clean-catch urine & time consuming (average waiting times of $30-70$ minutes), contamination rates of 5-27\% \\
Urine collection bags & high false-positive and contamination rates (85\%) \\
\hline
\end{tabular}

study of Kass in the 1950s that comprised adult asymptomatic and symptomatic women (4).

According to recent guidelines in infants and young children any bacterial growth in urine sample obtained by suprapubic aspiration confirms UTI $(5,6)$. There is a difference between European and American guidelines regarding bacterial number cut-off for UTI diagnosis. European guidelines consider that growth of $10.000 \mathrm{CFU} / \mathrm{mL}$ or even $1000 \mathrm{CFU} / \mathrm{mL}$ are sufficient for UTI diagnosis from a catheterized urine sample $(1,5)$, whereas United States and Canadian guidelines require the presence of at least $50.000 \mathrm{CFU} / \mathrm{mL}(3,6)$. It is recommended to remove the first few milliliters of urine drained by bladder catheterization in order to reduce the contamination rate (3).

Use of the lower criteria of $10.000 \mathrm{UFC} / \mathrm{ml}$ is important in pediatric population, as an infant or small child with urgency or frequent voiding is not able to hold urine long enough to allow bacterial multiplication to reach 50.000 or $100.000 \mathrm{CFU} / \mathrm{mL}$, leading to fewer diagnosed cases, and possible morbidities (4).

A new study suggest that reducing the bacteriologic criteria to diagnose a UTI in infants, toddlers and preschool children with fever or UTI symptoms plus leucocyturia from previous cut-off $>100.000 \mathrm{CFU} / \mathrm{mL}$ or $>50.000$ $\mathrm{CFU} / \mathrm{mL}$ to newer cut-off $>10.000 \mathrm{CFU} / \mathrm{mL}$ in an appropriately obtained urine sample would have no consequence on diagnostic specificity and sensitivity in accordance with the new European guidelines, but allow to recognize UTIs in this age population group (4).

A recent study concluded that dipstick examinations of clean-catch urine sample through standardized stimulation technique are a useful screening test in febrile infants younger than 3 months old for diagnosing UTIs and it may represent a good alternative to invasive method like bladder catheterization or suprapubic aspiration (7).

According to American Academy of Pediatrics (AAP) guidelines (2011) and revised in 2017, criteria for a true and definitive diagnosis of UTI are the presence of both quantitative urine culture and positive results on urinalysis (presence of white blood cells). A febrile UTI produces both fever or clinical symptoms as well as leucocyturia or pyuria (8).

Asymptomatic bacteriuria (ASB) is defined by significant bacteria count in urine specimen $(>100.000 \mathrm{UFC} / \mathrm{ml}$ or positive urine culture) in children with absence of any symptoms of UTI. ASB will have no pyuria, despite the positive urine culture. Pyuria is a hallmark of UTI (8). It occurs more frequently in preschool and school age girls. Incidence declines with increasing age. Because it does not cause kidney damage, screening and treatment for ASB should be discouraged (8).

In children with febrile UTIs complete cell blood counts and blood smear, acute phase reactants (the erythrocyte sedimentation rate, C-reactive protein, procalcitonin), blood culture when necessary, kidney function studies (creatinine, blood urea nitrogen) and serum electrolytes are recommended.

\section{Imaging studies}

Urinary tract ultrasound is recommended in all children with febrile UTI or recurrent lower or nonfebrile UTIs. Voiding cystourethrography (VCUG), to detect or rule out vesicoureteral reflux presence, is recommended in case of: recurrent UTIs, abnormal ultrasound, atypical UTI. Sometimes, in selected cases, other imaging studies may be necessary $(1,3,6)$.

\section{Treatment}

After urine sample has been obtained and, when necessary, blood samples collected, antibiotic therapy should be started as soon as possible. According to recent studies, early treatment is reported to reduce bacteraemia, improve clinical outcome and reduce the incidence of renal scar formation, while any delay in therapy initiation awaiting a new urine specimen for culture, as recommended, may have severe consequences (9-11). Empiric antibiotic treatment should be started for suspected UTI in a sick child, and if necessary, changed later according to sensitivity results for the isolated uropathogen. Guidelines recommend that empiric antibiotic treatment for suspected UTI should be 
based on local susceptibilities derived from available epidemiologic local information (1). Waiting for urine culture results, all patients will receive empirical antibiotics for at least 48-72 hours which can be changed later based on antibiotic sensitivity. Avoiding the use of antibiotics with known resistance and wide spectrum drugs, we may assist to a partial sensitivity recovery of uropathogens. A recent study confirmed that resistance pattern of the bacterial strains isolated from urine samples collected from routinely submitted outpatient and inpatient urine samples is different (12).

According to current guidelines, antibiotics should not be used in infants and children to treat asymptomatic bacteriuria, otherwise we may select resistant bacteria.

In infants and children older $\geq 3$ months with lower or nonfebrile UTI a short oral antibiotic course (3-5 days) is recommended (Trimethoprim/Sulfamethoxazole, Nitrofurantoin, $1^{\text {st }}$ or $2^{\text {nd }}$ generation Cephalosporin, Amoxicillin/clavulanic acid; and sometimes, based on local sensibility, even: Ampicillin, Amoxicillin), but not shorter than 1- 2 days. An old drug, Fosfomycin on oral route, widely used in adults, in pediatrics having just few published data, is recommended as a single 2-g dose for cystitis in children and adolescents. High urinary concentrations are observed in approximately 4 hours after drug intake that persist for several days. The dose may be repeated every 2-3 days for 3-7 times $(13,14)$.

Parenteral antibiotic therapy is recommended in case of: complicated UTI (kidney abnormalities, decreased kidney function, and sepsis), gastric intolerance, febrile UTI, urinary sepsis, noncompliance to oral treatment and in infants younger than 3 months old with UTI suspicion.

In febrile UTI antibiotic treatment is recommended for 10 to 14 days, at beginning with an intravenous antibiotic for 2 to 4 days then changed to oral antibiotics in case of good clinical evolution (if fever disappears, general state is improving, etc).

In infants and children with first UTI episode antibiotic prophylaxis should not be routinely recommended, but this may be considered in those with recurrent UTIs or if kidney abnormalities are present.

According to recent guidelines, infants and children who receive aminoglycosides (Amikacin, Tobramycin, Gentamicin), one single dose/day is recommended, this frequency being safer and equally effective as twice daily (1, 11). Also, Amikacin in a single intramuscularly dose/day could be a therapeutic option in case of suspected multidrug resistant (MDR) uropathogen as the UTI ethiological agent until the culture result is revealed. To prevent the oc- currence of aminoglycosides side-effects (nephro- and ototoxicity), Amikacin should be given as a total single daily dose of $15 \mathrm{mg} / \mathrm{kg}$ with a treatment length of 7 days (15).

Based on above mentioned data, the antibiotic choice in acute UTI treatment in outpatient children is illustrated in Table III.

\section{Discussion}

UTI can be the first sign in 30\% of children with urinary tract abnormalities. During the first 6-12 months after an initial UTI episode nearly $30 \%$ of infants and children, with urinary tract abnormality, namely vesicoureteral reflux, suffer recurrent UTIs (5).

UTI caused by resistant bacterial strains has an increasing prevalence in children. ESBL-producing gram-negative bacteria etiology in children UTIs represents an important therapeutic challenge. Another issue is represented by the growing percentage of UTIs caused by MDR pathogens for which there are limited therapeutic options.

Results of a recent meta-analysis highlights that in the pediatric population 14\% UTIs (1 out of 7) are caused by ESBL-producing Enterobacteriaceae (ESBL-PE). Risk factors for UTIs caused by ESBL-PE are: history of vesicouretral reflux, previous UTI as well as recent antibiotic therapy during the previous month or even in the last 6 months. These pathogens are associated with higher length of hospital stay, more than 1.5 times compared to other uropathogens, higher costs (more likely to be managed in inpatient settings) as well as the exposure of the patient to the hospital acquired infection risk (16).

The global spread of ESBL-PE among pediatric UTIs is different, the ESBL-PE UTIs rate being about $40 \%$ in Asia compared to Europe where this rate is close to $20 \%$ and 5\% in North America (16). This difference is assigned to local variability in antibiotic use as well as the enormous consumption of broad-spectrum cephalosporins as first line treatment, leading to the appearance of MDR bacteria.

ESBL-PE etiology leads to limited therapeutic options resulting in an important obstacle in the clinical treatment of UTIs (6). ESBL-PE are resistant to majority of betalactam antimicrobials, the therapeutic options being more restricted by the associated co-resistance to other antimicrobials. Beta-lactam-beta-lactamase inhibitor combinations, Aminoglycosides, Carbapenems and Tigecycline, represent alternative treatment options for ESBL-PE UTIs. The big disadvantage of all these drugs is that they have to be administered parenterally, so patients need to be treated as inpatients (16). Febrile children who fail to respond to antibiotherapy within 2-3 days are prone to kidney scar

Table III. Antibiotic options in case of lower versus febrile UTIs

\begin{tabular}{|c|c|c|}
\hline Diagnosis & First line & Alternative \\
\hline Lower UTI/acute cystitis & $\begin{array}{l}\text { Amoxicillin/clavulanic acid } \\
\text { Trimethoprim/sulfamethoxazole } \\
\text { Nitrofurantoin } \\
\text { Fosfomycin trometamol }\end{array}$ & $\begin{array}{l}1^{\text {st }} \text { or } 2^{\text {nd }} \text { generation of Cephalosporin (Cefuroxim, } \\
\text { Cephalexin) } \\
\text { Nitrofurantoin }\end{array}$ \\
\hline Febrile UTI & Amoxicillin/clavulanic acid & $\begin{array}{l}2^{\text {nd }} \text { or } 3^{\text {rd }} \text { generation Cephalosporin (Cefuroxim, Ceftibu- } \\
\text { ten, Cefpodoxim, etc) }\end{array}$ \\
\hline
\end{tabular}


formation, especially if they have a history of vesicoureteral reflux or recurrent UTIs. These findings are particularly important in pediatric population. To prevent long-term sequelae such as kidney scarring, high blood pressure and chronic kidney disease a precocious diagnosis and appropriate treatment of ESBL-PE and MDR uropathogen as UTIs ethiological agent is essential (16).

In the study conducted by Duffy et al. a time-related association between previous Trimethoprim-Sulfamethoxazole treatment and Escherichia coli resistant strains from urine specimens in children was found, with stronger associations for more recent Trimethoprim prescriptions (in the previous year) (17).

A very recent prospective study that included almost 4000 UTI samples (32\% pediatric patients) demonstrated that Fosfomycin is considerable active against both Grampositive and Gram-negative bacteria including resistant organisms like ESBL-PE (>90\%) and Methicillin-resistant Staphylococcus aureus (MRSA) (88\%) except for Acinetobacter spp. Fosfomycin, an old drug, may be an oral option in the era of MDR bacteria and should be kept in mind in pediatric UTIs treatment (2).

Given the lack of new antibiotics, there is a justifiable motivation to test older drugs that maintain some activity against MDR bacteria. Of these, Fosfomycin seems to be a promising choice because of its wide susceptibility against both Gram-positive and Gram-negative bacteria. Published microbiological data support this option, as susceptibility rate is very high in Klebsiella pneumoniae and, especially, in E. coli (18).

Data on antibiotic resistance patterns of Gram-negative organisms in Romania are incomplete. A recent study performed in central Romania, that included 107 small infants with UTI, concluded that there is a terrifying high antibiotic resistance rate of E. coli and Klebsiella spp. for Aminopenicillins, Ceftriaxone, Cefuroxime, Gentamicin and Ciprofloxacin, respectively. Almost $81 \%$ of the E. coli and $43 \%$ of the Klebsiella spp. isolates were ESBL-producers. The resistance rates of this 2 major uropathogens to Piperacillin/Tazobactam, Meropenem, Nalidixic acid, Chloramphenicol and Colistin were low (19). The resistance rate in this research was higher than that reported by European Centre for Disease Prevention and Control (20). Based on the latest European Union reports; Romania has a high resistance rate to antibiotics for E. coli and for Klebsiella pneumonie (table IV) while the highest resistance rate from Europe were reported for Pseudomonas spp. to Piperacillin \pm Tazobactam, Fluoroquinolones, Ceftazidime, Aminoglycosides, Carbapenems, or combined resistance to these drugs (20).

The results of a recent study that comprised 31.000 urine isolates showed that uropathogens resistance to many antibiotics was higher in the inpatient vs. outpatient (table V), (16). A recent meta-analysis stated that $\mathrm{Ni}$ trofurantoin represents the most appropriate therapeutic option as first line treatment for lower or nonfebrile UTI.
The same study underlined that some drugs commonly used in primary care, including Ampicillin or Amoxicillin and Trimethoprim, may have no effectiveness as firstline treatment (20). Also we should not be tempted to prescribe broad-spectrum second line antibiotics, such as Amoxicillin/Clavulanic acid, Cephalosporins and Fluoroquinolones (21).

In contradiction with previous data, another study demonstrated that most UTIs in preschool children retained susceptibility to Nitrofurantoin, Amoxicillin/Clavulanic acid and Cephalexin (86-98\%) and Trimethroprim (74\%) (22).

In a recent study, Polat and Kara proved the usefulness of once-daily intramuscular Amikacin in children with lower UTIs caused by ESBL-producing E. coli strains susceptible to this drug, without any oral therapy option, treated as outpatients (15).

However, when choosing an antibiotic for first line empirical treatment of UTI the main criteria should be the local prevalence of resistance to antibiotics smaller than $20 \%(21)$.

\section{Conclusion}

The UTI diagnosis in children should be based on clinical presentation, physical examination, urinalysis, cut-off of urine culture, methods of urine collection, inflammatory markers, and sometimes imaging studies. Prompt and appropriate diagnosis and treatment of a febrile UTI is important (in particular, in infants younger than 3 months). The significant incidence of MDR uropathogen strains in pediatric population should be taken into consideration in the attempt to suggest empiric treatment protocols. Nitrofurantoin and Fosfomycin, considered as old drugs, can represent alternatives for oral treatment and/or prophylaxis of UTIs in children or in case of resistance suspicion to

Table IV. Uropathogen resistance to antibiotics in Romania according to European Centre for Disease Prevention and Control report (20)

\begin{tabular}{lcc}
\hline Uropathogen & Drug & Resistance rate \\
\hline E. coli & Aminopenicillins & $72,3 \%$ \\
& Fluoroquinolones & $30,6 \%$ \\
& $3^{\text {rd }}$ generation Cephalosporins & $23,4 \%$ \\
Klebsiella & Aminoglycosides & $15 \%$ \\
pneumoniae & Carbapenems & $1 \%$ \\
& MDR & $11 \%$ \\
& Aminoglycosides & $68 \%$ \\
& Carbapenemes & $62 \%$ \\
& MDR & $31 \%$ \\
\hline
\end{tabular}

Table V. Uropathogen resistance to antibiotics in the inpatient vs. outpatient (16).

\begin{tabular}{lccc}
\hline Uropathogen & Drug & Inpatient & Outpatient \\
\hline E. coli & $\begin{array}{c}\text { Trimethoprim/ } \\
\text { Sulfamethoxazole }\end{array}$ & $30 \%$ & $24 \%$ \\
& Cephalothin & $22 \%$ & $16 \%$ \\
Klebsiella spp. & Cephalothin & $14 \%$ & $7 \%$ \\
Enterobacter spp. & Ceftriaxone & $24 \%$ & $12 \%$ \\
Enterococcus spp. & Ceftazidime & $33 \%$ & $15 \%$ \\
& Ampicillin & $13 \%$ & $3 \%$ \\
& Ciprofloxacin & $12 \%$ & $5 \%$ \\
\hline
\end{tabular}


other drug classes. This is probably due to the very scarce use of these drugs in the last years.

Assessment of the risk factors for MDR uropathogens is mandatory as it may help to choose appropriate empirical antibiotic therapy. If antibiotic exposure has been occurred in the preceding 3-6 months different antibiotic classes should be recommended for childhood UTI' treatment.

\section{Authors' contribution}

Carmen Duicu (Conceptualization; Investigation; Supervision; Writing -original draft; Writing - review \& editing) Iulia Armean (Data curation; Funding acquisition; Writing - original draft)

Cornel Aldea (Supervision; Validation; Writing - review \& editing)

\section{Conflict of interest}

The authors confirm that this article content has no conflict of interest.

\section{References}

1. NICE guideline. Urinary tract infection in under 16s: diagnosis and management (CG54). Updated 2017. https://www.nice.org.uk/guidance/ cg54

2. Patwardhan $\mathrm{V}$, Singh S. Fosfomycin for the treatment of drug-resistant urinary tract infections: potential of an old drug not explored fully. Int Urol Nephrol. 2017 Sep;49:1637-43.

3. AAP SUBCOMMITTEE ON URINARY TRACT INFECTION. Reaffirmation of AAP Clinical Practice Guideline: The Diagnosis and Management of the Initial Urinary Tract Infection in Febrile Infants and Young Children 2-24 Months of Age. Pediatrics. 2016 Dec;138(6): pii: e20163026.

4. Primak W, Bukowski T, Shuterland R, et al. What Urinary Colony Count Indicates a Urinary Tract Infection in Children? J Pediatr. 2017 Dec;191:259-61.

5. Stein R, Dogan HS, Hoebeke $P$, et al. Urinary tract infections in children: EAU/ESPU guidelines. Eur Urol. 2015 Mar;67(3):546-58.

6. Ammenti A, Cataldi L, Chimenz R, et al. Febrile urinary tract infections in young children: recommendations for the diagnosis, treatment and follow-up. Acta Paediatr. 2012 May;101(5):451-7.

7. Herreros ML, Tagarro A, García-Pose A, et al.. Performing a urine dipstick test with a clean-catch urine sample is an accurate screening method for urinary tract infections in young infants. Acta Paediatr. 2018 Jan;107(1):145-50.
8. Subcommittee on Urinary Tract Infection, Steering Committee on Quality Improvement and Management, Roberts KB. Urinary tract infection: Clinical practice guideline for the diagnosis and management of the initial UTI in febrile infants and children 2 to 24 months. Pediatrics 2011 Sep;128(3):595-610.

9. Coulthard MG, Lambert HJ, Vernon SJ, et al. Does prompt treatment of a urinary tract infection in preschool children prevent renal scarring: mixed retrospective and prospective audits. Arch Dis Child. 2014 Apr;99(4):3427.

10. Karavanaki KA, Soldatou A, Koufadaki AM, et al. Delayed treatment of the first febrile urinary tract infection in early childhood increased the risk of renal scarring. Acta Paediatr. 2017 Jan;106(1):149-54.

11. Stein R, Dogan HS, Hoebeke $P$, et al. Urinary tract infections in children: EAU/ESPU guidelines. Eur Urol. 2015 Mar;67(3):546-58.

12. Saperston KN, Shapiro DJ, Hersh AL, et al. A comparison of inpatient versus outpatient resistance patterns of pediatric urinary tract infection. J Urol. 2014 May;191(5 Suppl):1608-13.

13. Calzi A, Grignolo S, Caviglia I, et al. Resistance to oral antibiotics in 4569 Gramnegative rods isolated from urinary tract infection in children. Eur $J$ Pediatr. 2016 Sep;175(9):1219-25.

14. Hsu AJ, Tamma PD. Treatment of multidrug-resistant Gram-negative infections in children. Clin Infect Dis. 2014 May;58(10):1439-48.

15. Polat M, Kara SS. Once-daily intramuscular amikacin for outpatient treatment of lower urinary tract infections caused by extended-spectrum ? -lactamase-producing Escherichia coli in children. Infect Drug Resist. 2017 Nov; 10:393-399.

16. Flokas ME, Detsis M, Alevizakos M, et al. Prevalence of ESBL-producing Enterobacteriaceae in paediatric urinary tract infections: A systematic review and meta-analysis. J Infect. 2016 Dec;73(6):547-57.

17. Duffy MA, Hernandez-Santiago V, Orange G, et al. Trimethoprim prescription and subsequent resistance in childhood urinary infection: multilevel modelling analysis. Br J Gen Pract. 2013 Apr;63(609):e238-e243.

18. Mazzariol A, Bazaj A, Cornaglia G. Multidrug-resistant Gram-negative bacteria causing urinary tract infections: a review. J Chemother. 2017 Dec;29(sup1):2-9.

19. Falup-Pecurariu O, Leibovitz E, Bucur M, et al. High resistance rates to 2nd and 3rd generation cephalosporins, ciprofloxacin and gentamicin of the uropathogens isolated in young infants hospitalized with first urinary tract infection. Biomed Res. 2017;28(20):8774-8779

20. EARSS. Antimicrobial resistance surveillance in Europe 2016. https:// ecdc.europa.eu/sites/portal/files/documents/AMR-surveillanceEurope-2016.pdf

21. Bryce A, Hay AD, Lane IF, et al. Global prevalence of antibiotic resistance in paediatric urinary tract infections caused by Escherichia coli and association with routine use of antibiotics in primary care: systematic review and meta-analysis. BMJ. 2016 Mar;352:1939.

22. Butler CC, O'Brien K, Wootton M, et al. Empiric antibiotic treatment for urinary tract infection in preschool children: susceptibilities of urine sample isolates. Fam Pract. 2016 Apr;33(2):127-32. 